\title{
Ultrastructural Analysis of a Unique Malignant Brenner Tumor
}

\author{
M.P. Goheen, ${ }^{*}$ L.M. Roth, ${ }^{*}$ and J.R. Broshears ${ }^{* *}$ \\ * Dept. of Pathology. Indiana University School of Medicine, Indianapolis, IN 46202 \\ ${ }^{* *}$ Bradenton Pathology, Bradenton, FL 34205
}

Brenner tumors account for 1-2\% of all ovarian tumors, and most cases are considered to be of a surface epithelial-stromal origin $\{1\}$. The epithelial component of the tumor consists of nests of transitional cells similar to those lining the urinary bladder. An 85 year old woman presented with a right lower quadrant mass. A right oophorectomy was performed, and the ovary was completely replaced by tumor. Histologic examination showed both benign and malignant nests of Brenner tumor, and argyrophilic cells were identified. Although argyrophilic cells have been described in ovarian Brenner tumor and urinary bladder epithelium, transition to trabecular carcinoid was a unique finding. Examination of the tumor with transmission electron microscopy (TEM) was performed to provide additional information about this rare tumor.

Tissue retrieved from formalin was fixed in 3\% buffered glutaraldehyde, post fixed in 1\% $\mathrm{OsO}_{4}$, and processed for TEM using standard methodology. $1 \mu$ thick sections stained with toluidine blue were examined and appropriate areas were identified and sectioned for TEM. The sections were stained with 3\% uranyl acetate aq. and Reynolds lead citrate. Sections of tumor were examined using an FEI Tecnai G $^{2}$ Spirit Bio (TWIN) electron microscope operated at $80 \mathrm{Kv}$. Images were captured with an AMT XR-60 digital camera.

Three distinct components of this Brenner tumor were identified. The cells of benign Brenner tumor had uniform nuclei with nuclear grooves and occasional distinct nucleoli; the cytoplasm contained granular endoplasmic reticulum, mitochondria, and free ribosomes along with cell junctions and intercellular filopodia. Cystic areas of this benign component had a microvillous surface and numerous mucin granules indicative of mucinous metaplasia. Some of these cells also contained a few round neurosecretory granules. The malignant component was easily identified by its large nuclei with irregular contours. The trabecular carcinoid component consisted of small cells with uniform round to oval nuclei having irregularly clumped chromatin and cytoplasm with numerous neurosecretory-type round granules ranging in size from 200 to $400 \mathrm{~nm}$. The granules observed by electron microscopy in the Brenner tumor and trabecular carcinoid are consistent with those described in forgut-hindgut carcinoids and are distinctly different from the pleomorphic granules of midgut carcinoid $\{2\}$.

We are not aware of any cases of malignant Brenner tumor associated with carcinoid in the literature. We interpret this case as a low grade malignant Brenner tumor that evolved into a trabecular carcinoid, and an example of neuroendocrine transformation of a common epithelial tumor. 
References

\{1\} R.E. Scully et al. Tumors of the ovary, AFIP (1998) 153.

$\{3\} \quad$ W.C. Black III. Lab Invest (1968) 19:473-86.
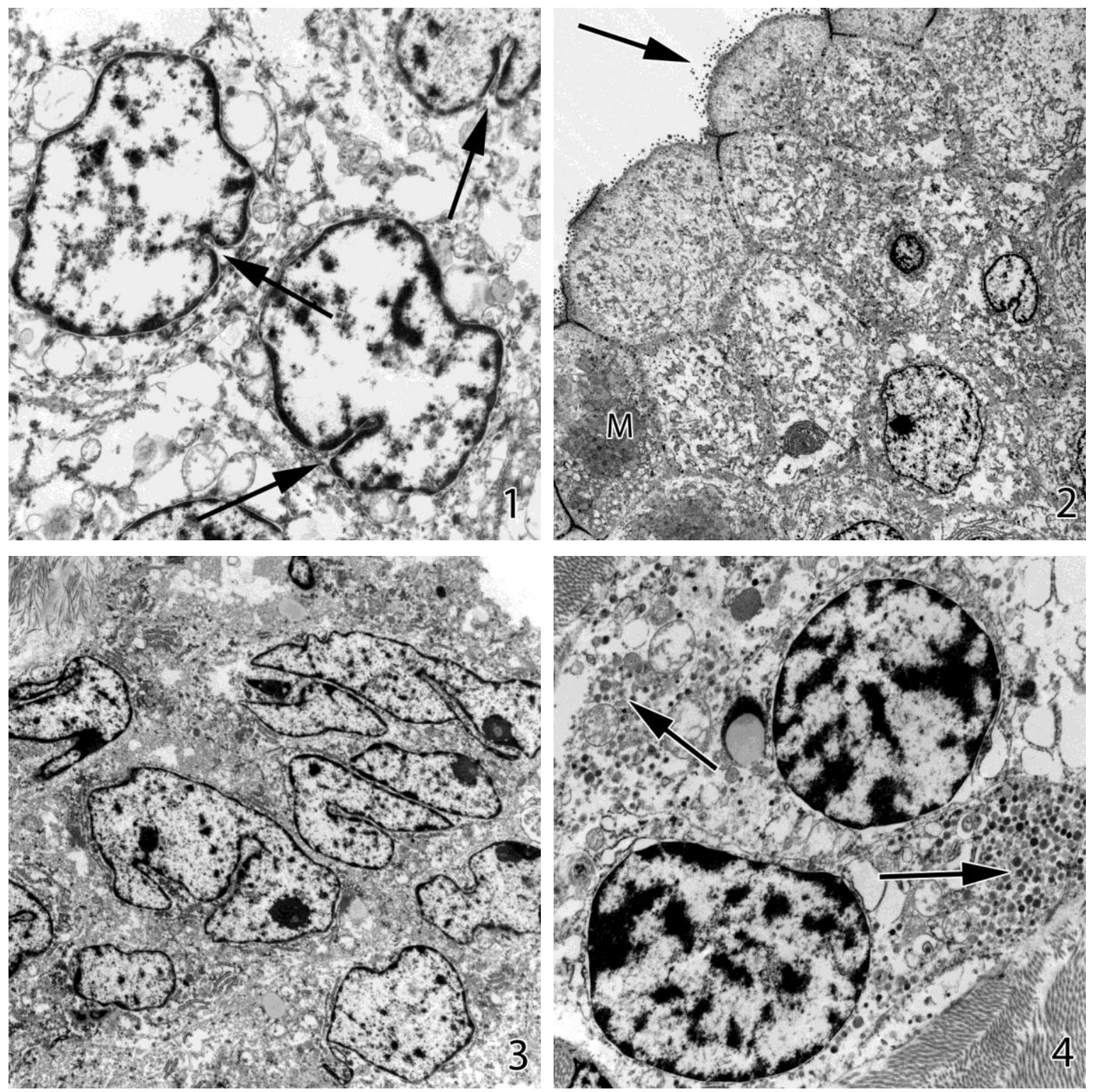

Fig 1, Benign Brenner tumor cells displaying nuclear grooves (arrows). 7,750X

Fig 2, Cystic region of benign Brenner tumor $(\mathrm{M})$ mucin granules, surface microvilli (arrow). 2,500X

Fig 3, Irregular contoured nuclei of malignant Brenner tumor. 4,000X

Fig 4, Trabecular carcinoid displaying round nuclei with irregularly clumped chromatin and numerous neurosecretory granules (arrows). 6,600X 